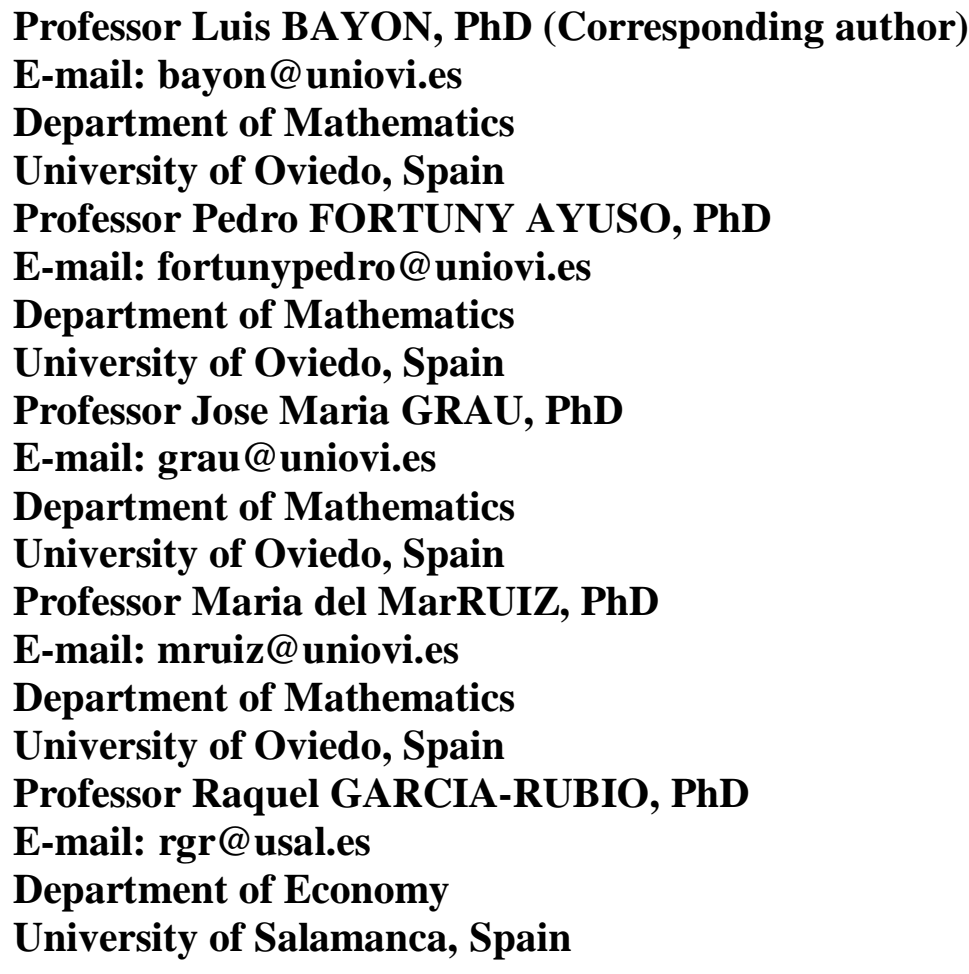

\title{
AN OPTIMAL STOPPING PROBLEM FOR AN INVESTING FIRM
}

Abstract. The information on the costs, benefits and opportunities is an essential tool for the study of real options in firms that make large investments. We study investment decisions regarding disruptive economies. The real options logic is unsuitable for single-investment events, which are becoming frequent in this type of economies. We propose a strategy for a firm seeking to invest in the very best technology among a sequence of opportunities. If the choice is not the best, the invested capital will be lost. The yield will depend on whether the chosen option is the best and on the investment. Our analysis shows that the optimal strategy for the investing firm is to invest a specific capital in the relatively best option after a threshold which depends on the number of opportunities.

Keywords: Stopping problem; Secretary problem; Investing firm; Utility function; Decisions.

JEL Classification: C44, D21 
Luis Bayón, Pedro Fortuny Ayuso, Jose Maria Grau, Maria del Mar Ruiz, Raquel García-Rubio

\section{Introduction}

Numerical and computational techniques in Economics [13, 14, 15, 17, 18] are nowadays of foremost importance both in general policy development and in firms' financial planning. Real Options, which have become an essential tool in the last years for firms facing major investments [4, 6], are especially suited to multistaged scenarios in which information on the costs, benefits and opportunities is complete (in a rough sense). They are modeled as processes in which significant investments take place discretely and where decisions to invest can be delayed (or definitely disregarded) and, stochastically, they tend to require a Poisson processes (in contrast to the classical Financial Options theory where just Brownian motion is the basic theoretical tool $[2,5,12])$.

As shown by (Arikan et al, 2002), the Real Options logic is unsuitable for single-investment events of the type "winner-takes-all", which are becoming frequent in modern disruptive economies (v.gr. choosing between Google or the other different search engines in the late 1990s, or the buy of Whatsapp by Facebook in 2014) or in the development of pharmaceutical compounds, where costs are so high that starting research on one may prevent the development of a different one.

In this regard, [1] presents a model broadly derived from the well-known "Secretary Problem", in which an actor has to choose the best among $n$ different items presented to him sequentially and at each step he has information only on the items already presented. This problem has a very elegant solution: Dynkin (1963) and Lindley (1961) independently proved that the best strategy is a so-called threshold strategy. The secretary problem has been studied by many authors in different contexts: applied probability, statistics, decision theory. Extensive bibliographies on the topic can be found in [8], [9] and [16].

In this paper we state a related problem: an Investing Firm (IF) with an available capital is sequentially presented with a different investment opportunity and its aim is to choose the best among them and the optimal investment to make. The IF can only either reject the present opportunity or choose it (rejected ones are discarded for ever) and no more investments are made after choosing one. One of the main features of this model is that if the chosen opportunity is the very best overall, then the IF will receive a return based upon its investment, but it will receive 0 otherwise and the invested capital is lost.

In contrast to [1], we study in depth the problem for different utility functions, showing how there is a decision threshold (discarding $n$ opportunities and then choosing the first one which is the overall best after that threshold) which changes depending on the utility function. We also study the asymptotic properties of our model. 
An Optimal Stopping Problem for an Investing Firm

\section{Statement of the problem}

The investing problem we shall study is properly described as follows (IF is the investment firm):

- The IF is faced with a sequence of $n>0$ pairwise independent investment opportunities (IO).

- The IF can invest in only one IO.

- The IOs are presented to the IF in a random order.

- The IF has a capital $w$ (wealth) to invest. After normalization, we may assume $w=1$.

- The IF can only know the relative rank of each IO at the time it is presented.

- The IOs can be ordered without ties.

- The decision to choose a specific IO is based only on the relative ranks of the IOs considered so far.

- A rejected IO cannot be recalled later.

- The initial information of the IF is only the number $n$ of IOs.

- The IF must decide whether to invest a certain amount $x$ in the chosen opportunity, keeping $1-x$ to itself, or not to invest at all.

- If after all projects are observed, the chosen opportunity is the overall best, then the return on the investment is given by $y=R x$; where $R>0$ is a known rate of return. Otherwise, the IF loses the invested capital $x$ (so that $y=0$ ).

- No interest accrues on uninvested capital.

- There is a fixed and equal cost for each IO analyzed.

The aim of the IF is to maximize the utility $U(x)$ (not just the final wealth): we shall study several utility functions (instead of simply dealing with the capital gain).

\section{Preliminary results}

The stated problem is an optimal stopping problem (OSP) whose payoff depends on observing the relative ranks (and not the actual values) of the IOs. It has the following solution: there is an integer $r \geq 1$ such that the IF must reject the first $r-1$ IOs. Afterwards, the IF must chose the next IO which is relatively better than all the previous ones. However, $r$ depends on the utility function. We also study the asymptotic properties of the solution when $n \rightarrow \infty$.

\subsection{Asymptotic results}

The following are some technical results which we shall use extensively in forthcoming sections. 
Luis Bayón, Pedro Fortuny Ayuso, Jose Maria Grau, Maria del Mar Ruiz, Raquel García-Rubio

Proposition 1. Let $\left\{F_{n}\right\}_{n \in \mathbb{N}}$ be a sequence of real functions such that $F_{n}(k) \in\{0, \ldots, n\}$. Let $\mathcal{M}(n)$ be the minimum value for which $F_{n}$ is maximum. Assume that the sequence of functions $\left\{f_{n}\right\}_{n \in \mathbb{N}}$ given by $f_{n}(x):=F_{n}(\lfloor n x\rfloor)$ converges uniformly on $[0,1]$ to a function $f$ continuous in $[0,1]$ having a single global maximum $\theta \in[0,1]$. Then:

1. $\lim _{n} \mathcal{M}(n) / n=\theta$.

2. $\lim _{n} F_{n}(\mathcal{M}(n))=f(\theta)$.

3. If $\mathfrak{M}(n) \sim \mathcal{M}(n)$ then $\lim _{n} F_{n}(\mathfrak{M}(n))=f(\theta)$.

Proof. The proof is straightforward and analogous to that presented in [3] for functions $F_{n}$ of a real variable and domain $[0, n]$ and $f_{n}(x)=F_{n}(n x)$.

Under the assumption of the uniform convergence of the sequence $\left\{f_{n}\right\}$ to a continuous function, the following result shows that the limit function $f$ can be easily found provided that the functions $F_{n}$ are defined by a backwards recursion.

Theorem 1 Let $s_{n} \rightarrow s$ be a convergent sequence of real numbers. Let a sequence of functions $F_{n}:\left[s_{n}, n\right] \cap \mathbb{Z} \rightarrow \mathbb{R}$, be defined recursively by the conditions:

$$
F_{n}(k)=G_{n}(k)+H_{n}(k) F_{n}(k+1) \text { and } F_{n}(n)=\mu
$$

Set $f_{n}(x):=F_{n}(\lfloor n x\rfloor), \quad h_{n}(x):=n\left(1-H_{n}(\lfloor n x\rfloor)\right) \quad$ and $\quad g_{n}(x):=$ $n G_{n}(\lfloor n x\rfloor)$. If both $h_{n}(x)$ and $g_{n}(x)$ converge on $(s, 1)$ and uniformly on $[s+$ $\left.\varepsilon, \varepsilon^{\prime}\right]$ for all $0<\varepsilon<\varepsilon^{\prime}<1$ to functions $h(x)$ and $g(x)$ (resp.), both continuous in $(s, 1)$, and $f_{n}(x) \rightarrow f(x)$ uniformly on $[s, 1]$ with $f \in \mathcal{C}[s, 1]$, then $f(1)=\mu$ and the following equality holds in $(s, 1)$

$$
f^{\prime}(x)=f(x) h(x)-g(x)
$$

Proof. Just proceed as in [10].

\section{The Solution}

Let $\mathcal{U}: \mathbb{R} \mapsto \mathbb{R}$ be the utility function of the IF. We fix an investable normalized capital $w=1$, part of which can be invested on an IO with success probability $p$, which in case of success yields an return rate $R>0$ (as a consequence, there is a probability $1-p$ of losing all the invested capital). If the IF invests $x \in[0,1]$, the expected utility is:

$$
\mathcal{U}_{p}(x)=p \cdot \mathcal{U}(1+R x)+(1-p) \cdot \mathcal{U}(1-x)
$$

Given $R$, define $\mathfrak{m}(p)$ as the optimal investment (when it is unique) for each value of $p$ as:

$$
\mathfrak{m}(p):=\arg \max _{x \in[0,1]} u_{p}(x)
$$
unique) is:

Thus, the expected utility after the optimal investment (assuming it is

DOI: 10.24818/18423264/54.1.20.05 
An Optimal Stopping Problem for an Investing Firm

$$
\Psi(p):=U_{p}(\mathfrak{m}(p))=\max _{x \in[0,1]} u_{p}(x)
$$

Remark 1.If $\mathcal{U}^{\prime \prime}(x)>0$ for all $x$, then $\mathcal{U}_{p}^{\prime \prime}(x)>0$ and $\mathfrak{m}(p) \in\{0,1\}$ : that is, the IF invests either all or nothing. This is what happens under risk-neutrality or risk-affection.

Taking into account that choosing the $r$-th opportunity which is better than the previous ones, the probability that it is the best overall is $r / n$, the dynamic equation of the problem is:

$$
\mathbb{U}_{n}(r)=\frac{1}{r+1} \max \left[\Psi\left(\frac{r+1}{n}\right), \mathbb{U}_{n}(r+1)\right]+\frac{r}{r+1} \mathbb{U}_{n}(r+1) ; \mathbb{U}_{n}(n)=\mathcal{U}(1)
$$

where $\mathbb{U}_{n}(r)$ is the final expected utility after rejecting the $r$-th opportunity. The expected utility is $\mathbb{U}_{n}(0)$. The optimal strategy consists in rejecting each opportunity as long as it is better to pass on to the next one than to invest in the present one, that is:

$$
\Psi\left(\frac{r}{n}\right)<\mathbb{U}_{n}(r+1)
$$

Proposition 2.If $n$ is the number of opportunities, there exists $k_{n}$ such that the following strategy is optimal:

1. Reject the first $k_{n}$ opportunities.

2. After that, choose the first which is better than all the previous ones.

It is natural to ask what the asymptotic thresholds $k_{n}$ and utilities are. To this end, it is worth using an auxiliary function $\bar{U}(r)$ representing the expected utility when $r$ is taken as threshold. This function is defined by the following recurrence:

$$
\bar{U}_{n}(r)=\frac{1}{r+1} \cdot \Psi\left(\frac{r+1}{n}\right)+\frac{r}{r+1} \cdot \bar{U}_{n}(r+1) ; \bar{U}_{n}(n)=\mathcal{U}(1)
$$

For the sake of clarity, let us pose a simplified example, where the utility function is the identity $U(x)=x$. This gives a variant of the secretary problem where the payback is $R$ in case of success, -1 in case of error and 0 when no opportunities are chosen.

Example. Take $\mathcal{U}(x)=x$ as the utility function. Using the previous Theorem and Proposition, we can compute the optimal threshold solving the differential equation:

whose solution is:

$$
-1-R+\frac{y(x)}{x}=y^{\prime}(x) ; y(1)=1
$$

so that if $\mathbf{r}_{n}$ is the optimal threshold, then:

$$
y(x):=-(x(-1+(1+R) \log (x)))
$$

DOI: $10.24818 / 18423264 / 54.1 .20 .05$ 
Luis Bayón, Pedro Fortuny Ayuso, Jose Maria Grau, Maria del Mar Ruiz, Raquel García-Rubio

$$
\lim _{n \rightarrow \infty} \frac{\mathbf{r}_{n}}{n}=\vartheta
$$

where $\vartheta=e^{-\left(\frac{R}{1+R}\right)}$ is the maximum value of $y(x)$ in $[0,1]$.

The expected utility is:

$$
y(\vartheta)=e^{-1+\frac{1}{1+R}(1+R)}
$$

In particular, for $R=1$ :

$$
\vartheta=\frac{1}{\sqrt{e}}=0.6065 \ldots ; y(\vartheta)=\frac{2}{\sqrt{e}}=1.21306 \ldots
$$

which means that the optimal strategy for a large number of opportunities consists in rejecting each of them until the $60.65 \%$ has been seen and, afterwards, choosing the first which is relatively better than all the preceding ones.

With this strategy, the expected final capital is $1.21306 \ldots$ or, what amounts to the same, there is an expected benefit of $21.30 \%$.

\section{Utility Functions}

We study at this point the optimal strategy under different utility functions. Notice that at some points we assume that some functions $\mathbf{U}_{n}(x)$ converge to some other like in Theorem 1. Numerical computations lead us to conclude that this assumption is reasonable despite our inability (up to date) to prove it formally.

\subsection{The Square Root Utility Function}

First, we consider the square root:

$$
\mathcal{U}(x)=\sqrt{x}
$$

Under the conditions of our problem, the expected utility after investing a (normalized) capital $x$ is:

$$
u_{p}(x)=p \cdot \sqrt{1+R x}+(1-p) \cdot \sqrt{1-x}
$$

The optimal investment $m(p)$ is unique and is given by:

$$
\mathfrak{m}(p)=\left\{\begin{array}{lll}
\frac{-1+2 p-p^{2}+p^{2} R^{2}}{R\left(1-2 p+p^{2}+p^{2} R\right)} & \text { if } & \mathrm{p} \in\left[\frac{1}{1+\mathrm{R}}, 1\right] \\
0 & \text { if } & p \in\left[0, \frac{1}{1+R}\right]
\end{array}\right.
$$

Using both expressions, we get the expected utility for the optimal investment for $p>\frac{1}{1+R}$ :

$$
\Psi(p):=\mathcal{U}_{p}(\mathfrak{m}(p))=(1-p) \sqrt{\frac{(-1+p)^{2}(1+R)}{R(1+p(-2+p+p R))}}+\mathrm{p} \sqrt{\frac{p^{2} R(1+R)}{1+p(-2+p+p R)}}
$$

whereas for $p<\frac{1}{1+R}$ the optimal behavior is not to invest at all. Using now the recursive equation defining the expected utility for threshold $r$ : 


$$
\bar{U}_{n}(r)=\frac{1}{r+1} \cdot \Psi\left(\frac{r+1}{n}\right)+\frac{r}{r+1} \cdot \bar{U}_{n}(r+1) ; \bar{U}_{n}(n)=\mathcal{U}(1)
$$

and assuming that the functions $\mathbf{U}_{n}(x):=\bar{U}_{n}(\lfloor n x\rfloor)$ converge uniformly on $\left[\frac{1}{1+R}, 1\right]$ to the continuous and derivable function $y(x)$ on $\left(\frac{1}{1+R}, 1\right)$ with:

taking

$$
G_{n}(r)=\frac{1}{r+1} \Psi\left(\frac{r+1}{n}\right) \text { and } H_{n}(r)=\frac{r}{r+1}
$$

$$
h_{n}(x):=n\left(1-H_{n}(\lfloor n x\rfloor)\right) \text { and } g_{n}(x):=n G_{n}(\lfloor n x\rfloor)
$$

we have that the functions $g_{n}(x)$ converge on $\left(\frac{1}{R+1}, 1\right)$ to:

$$
\begin{gathered}
g(x):=(-1+x) \sqrt{\frac{1+R}{R-2 R x+R(1+R) x^{2}}}+\frac{(1-x) \sqrt{\frac{1+R}{R-2 R x+R(1+R) x^{2}}}}{x} \\
\quad+x \sqrt{\frac{R(1+R)}{1+x(-2+x+R x)}}
\end{gathered}
$$

and the $h_{n}(x)$ converge on $\left(\frac{1}{R+1}, 1\right)$ to:

$$
h(x):=\frac{1}{x}
$$
equation:

By Theorem 1, $y(x)$ satisfies in $\left(\frac{1}{R+1}, 1\right)$ the following differential

$$
y^{\prime}(x)=y(x) h(x)-g(x)
$$

with the condition $y(1)=\mathcal{U}(1)$. The solution of this differential equation gives:

where:

$$
\begin{aligned}
y(x) & =x-\sqrt{1+R} x+\sqrt{\frac{1+R}{R}} A+\sqrt{\frac{1+R}{R}} x \log \left(\frac{-\sqrt{R} x}{-1+x-A}\right) \\
& +\frac{(1+R)}{\sqrt{R}} x \log \left(\frac{R \sqrt{1+R}+\sqrt{R}(1+R)}{\sqrt{1+R}(-1+(1+R) x)+(1+R) A}\right)
\end{aligned}
$$

$$
A=\sqrt{1-2 x+(1+R) x^{2}}
$$

Now, using Proposition 1, we can compute the asymptotic values of the optimal threshold and expected utility. If $\mathbf{r}_{n}$ is that optimal threshold, then:

$$
\lim _{n \rightarrow \infty} \frac{\mathbf{r}_{n}}{n}=\vartheta
$$

where $\vartheta$ is the maximum value of $y(x)$ in $\left[\frac{1}{1+R}, 1\right]$ and $y(\vartheta)$ the expected utility.

Table 1 shows the values of the optimal threshold and expected utility for different rates $R$. When the number of opportunities is large, the optimal strategy

\section{DOI: $10.24818 / 18423264 / 54.1 .20 .05$}


Luis Bayón, Pedro Fortuny Ayuso, Jose Maria Grau, Maria del Mar Ruiz, Raquel García-Rubio

consists in rejecting each one until having seen $100 \cdot \vartheta_{R} \%$ of the total number $n$. Afterwards, the IF chooses the first one which is relatively better than all the previous ones. The expected utility is $y\left(\vartheta_{R}\right)$. For instance, for $R=1$, the expected utility is 1.06291277 whereas for $R=2$ it is 1.14760013 . Notice that for extremely large values of $R$, the threshold approaches $1 / e$, which is the threshold in the standard Secretary Problem.

Table 1. Square root utility function

\begin{tabular}{|c|c|c|}
\hline$R$ & $\vartheta_{R}$ & $y\left(\vartheta_{R}\right)$ \\
\hline $1 / 2$ & 0.76873644 & 1.02317255 \\
\hline $3 / 4$ & 0.71688191 & 1.04230022 \\
\hline 1 & 0.68012743 & 1.06291277 \\
\hline $3 / 2$ & 0.63081156 & 1.10542974 \\
\hline $5 / 3$ & 0.61874166 & 1.11959040 \\
\hline 2 & 0.59874122 & 1.14760013 \\
\hline $10^{6}$ & 0.36824710 & 368.24782757 \\
\hline
\end{tabular}

\subsection{The Logarithmic Utility Function}

We consider now the natural logarithm as a utility function:

$$
u(x)=\log (1+x)
$$

The expected utility after an investment of capital $x$ is:

$$
u_{p}(x)=p \cdot \log (2+R x)+(1-p) \cdot \log (2-x)
$$

so that the optimal investment $\mathfrak{m}(p)$ is unique and is given by:

$$
\mathfrak{m}(p)=\left\{\begin{array}{lll}
\frac{2(-1+p+p R)}{R} & \text { if } & p \in\left[\frac{1}{1+\mathrm{R}}, 1\right] \\
0 & \text { if } & p \in\left[0, \frac{1}{1+R}\right]
\end{array}\right.
$$

For values $p<\frac{1}{1+R}$ the optimal investment is not to invest. For $p>\frac{1}{1+R}$, the expected utility is:

$$
\Psi(p):=\mathcal{U}_{p}(\mathfrak{m}(p))=p \log (2 p(1+R))-(-1+p) \log \left(\frac{-2(-1+p)(1+R)}{R}\right)
$$

The recursive equation for the expected utility using threshold $r$ is:

$$
\bar{U}_{n}(r)=\frac{1}{r+1} \cdot \Psi\left(\frac{r+1}{n}\right)+\frac{r}{r+1} \cdot \bar{U}_{n}(r+1) ; \bar{U}_{n}(n)=U(1)
$$

Assuming that $\mathbf{U}_{n}(x):=\bar{U}_{n}(\lfloor n x\rfloor)$ converges uniformly on $\left[\frac{1}{1+R}, 1\right]$ to a continuous and differentiable function $y(x)$ on $\left(\frac{1}{1+R}, 1\right)$ and proceeding as in the previous section, we get that $g_{n}(x)$ converges on $\left(\frac{1}{R+1}, 1\right)$ to:

$$
g(x):=\left(-1+\frac{1}{x}\right) \log \left(\frac{2(1+R)(1-x)}{R}\right)+\log (2(1+R) x)
$$

DOI: 10.24818/18423264/54.1.20.05 
and $h_{n}(x)$ converges on $\left(\frac{1}{R+1}, 1\right)$ to:

$$
h(x):=\frac{1}{x}
$$

Theorem 1, guarantees that $y(x)$ satisfies, in $\left(\frac{1}{R+1}, 1\right)$, the following differential equation:

$$
y^{\prime}(x)=y(x) h(x)-g(x)
$$

with $y(1)=\mathcal{U}(1)$. From these conditions, it follows that:

$$
\begin{aligned}
y(x)= & \frac{1}{6}\left(\pi^{2} x+6 x \log (2)+3 x \log (2)^{2}+6 x \log (2) \log (1+R)\right) \\
& \left.+3 x \log (1+R)^{2}-6 x \log \left(\frac{2(1+R)}{R}\right)+6 x \log (x)\right) \\
& -6 x \log (1-x)(1+\log (x))-3 x \log (2(1+R) x)^{2} \\
+ & \left.6 \log \left(\frac{2(1+R)(1-x)}{R}\right)(1+x \log (x))-6 x \operatorname{PolyLog}(2, x)\right)
\end{aligned}
$$

where:

$$
\operatorname{PolyLog}(2, x)=\int_{0}^{x} \frac{-\log (1-t)}{t} d t
$$

The computation of the optimal threshold $\vartheta$ and the maximum expected utility, $y(\vartheta)$ is carried out using Proposition $1 ; \vartheta$ is the maximum value of $y(x)$ en $\left[\frac{1}{1+R}, 1\right]$.

Table 2 provides their values for some values of $R$.Considering that the if the IF does not invest anything at all, it ends up with the starting capital $w$ (i.e. an expected utility of $\log (2)=0.69314718 \ldots)$, we remark how, following the optimal strategy, if the number of opportunities is large, the IF would obtain for $R=1 / 2$ an expected utility of 0.72505881 , while for $R=1$ it would be 0.77459118 and for $R=5 / 3,0.83824206$. Notice how the increase in the expected utility with respect to $R$ is larger than that of the previous utility function.

Table 2. Logarithmic utility function

\begin{tabular}{|c|c|c|}
\hline$R$ & $\vartheta_{R}$ & $y\left(\vartheta_{R}\right)$ \\
\hline $1 / 2$ & 0.78149444 & 0.72505881 \\
\hline $3 / 4$ & 0.73327571 & 0.74952131 \\
\hline 1 & 0.69900717 & 0.77459118 \\
\hline $3 / 2$ & 0.65280685 & 0.82297146 \\
\hline $5 / 3$ & 0.64145138 & 0.83824206 \\
\hline
\end{tabular}

\section{DOI: $10.24818 / 18423264 / 54.1 .20 .05$}


Luis Bayón, Pedro Fortuny Ayuso, Jose Maria Grau, Maria del Mar Ruiz, Raquel García-Rubio

\subsection{The Exponential Utility Function}

Consider the utility function given by:

$$
\mathcal{U}(x)=1-e^{-t x}
$$

For this $U(x)$, we have

$$
u_{p}(x)=p \cdot\left(1-e^{-t(1+R x)}\right)+(1-p) \cdot\left(1-e^{-t(1-x)}\right)
$$

and the optimal investment $\mathrm{m}(p)$ is unique and is given by:

$$
\mathfrak{m}(p)=\left\{\begin{array}{lll}
-\frac{1}{(1+R) t} \log \left(\frac{t-p t}{p R t}\right) & \text { if } & \mathrm{p} \in\left[\frac{1}{1+\mathrm{R}}, 1\right] \\
0 & \text { if } & p \in\left[0, \frac{1}{1+R}\right]
\end{array}\right.
$$

The expected utility of the optimal investment for $p>\frac{1}{1+R}$ is:

$$
\Psi(p):=\mathcal{U}_{p}(\mathfrak{m}(p))=1+\frac{(-1+p)(1+R)}{e^{t}\left(\frac{1-p}{p R}\right)^{\frac{1}{1+R}} R}
$$

And the recurrence equation for the expected utility using threshold $r$ is:

$$
\bar{U}_{n}(r)=\frac{1}{r+1} \cdot \Psi\left(\frac{r+1}{n}\right)+\frac{r}{r+1} \cdot \bar{U}_{n}(r+1) ; \bar{U}_{n}(n)=\mathcal{U}(1)
$$

Using Theorem 1 as before, we obtain $g_{n}(x), g(x), h_{n}(x), h(x)$ and $y(x)$ such that

- $g_{n}(x)$ converge in $\left(\frac{1}{R+1}, 1\right)$ to: $g(x):=\frac{R+e^{-t}(1+R)(-1+x)\left(\frac{1-x}{R x}\right)^{-\frac{1}{1+R}}}{R x}$

- $h_{n}(x)$ converge in $\left(\frac{1}{R+1}, 1\right)$ to: $h(x):=\frac{1}{x}$

- $y(x)$ satisfies in $\left(\frac{1}{R+1}, 1\right)$ the following differential equation:

together with $y(1)=\mathcal{U}(1)$.

$$
y^{\prime}(x)=y(x) h(x)-g(x)
$$

Solving the differential equation, we obtain:

$$
\begin{aligned}
y(x) & =\frac{R\left(e^{t}-x\right)}{e^{t} R} \\
& +\frac{(1+R)\left(x^{\frac{R}{1+R}}(R x)^{\frac{1}{1+R}} \operatorname{Beta}\left(x,-1+\frac{1}{1+R}, 2-\frac{1}{1+R}\right)+\pi R^{\frac{1}{1+R}} x \csc \left(\frac{\pi}{1+R}\right)\right)}{e^{t} R}
\end{aligned}
$$

Using Proposition 1, we can compute the optimal threshold $\vartheta$ (as $\vartheta$ is the maximum value of $y(x)$ in $\left[\frac{1}{1+R}, 1\right]$ ), and the value of the maximum expected utility, $y(\vartheta)$. 
An Optimal Stopping Problem for an Investing Firm

Table 3. Exponential utility function

\begin{tabular}{|c|c|c|c|}
\hline & $R$ & $\vartheta_{R, t}$ & $y\left(\vartheta_{R, t}\right)$ \\
\hline \multirow{5}{*}{$t=1$} & $1 / 2$ & 0.53848590 & 0.644381 \\
\cline { 2 - 4 } & 1 & 0.70729857 & 0.665228 \\
\cline { 2 - 4 } & $3 / 2$ & 0.65423520 & 0.678225 \\
\cline { 2 - 4 }$t=2$ & 2 & 0.61767078 & 0.688099 \\
\hline \multirow{5}{*}{$t=5$} & $1 / 2$ & 0.53848590 & 0.869175 \\
\cline { 2 - 4 } & 1 & 0.70729857 & 0.876844 \\
\cline { 2 - 4 } & $3 / 2$ & 0.65423520 & 0.881625 \\
\cline { 2 - 4 } & 2 & 0.61767078 & 0.885258 \\
\hline \multirow{5}{*}{$t=10$} & $1 / 2$ & 0.53848590 & 0.993487 \\
\cline { 2 - 4 } & 1 & 0.70729857 & 0.993868 \\
\cline { 2 - 4 } & $3 / 2$ & 0.65423520 & 0.994106 \\
\cline { 2 - 4 } & 2 & 0.61767078 & 0.994287 \\
\cline { 2 - 4 } & $1 / 2$ & 0.53848590 & 0.999956 \\
\cline { 2 - 4 } & $3 / 2$ & 0.70729857 & 0.999959 \\
\cline { 2 - 4 } & 2 & 0.65423520 & 0.99996 \\
\hline
\end{tabular}

Table 3 gives a sample of values for the threshold and the expected utility for different values of $R$ and the parameter $t$. Notice how the optimum threshold is the same for fixed $R$, regardless of $t$.

\subsection{The Iso-Elastic Utility Function}

Finally, we consider the isoelastic utility function, the only class of utility functions with constant risk aversion.

$$
U(x)= \begin{cases}\frac{x^{t}-1}{t} & \text { for } t<1, t \neq 0 \\ \log (x) & \text { the limiting case for } t=0\end{cases}
$$

Notice that both the square root function $(t=1 / 2)$ and the logarithmic function, already covered, are members of this class. Isoelasticity models constant relative risk aversion (i.e. decision making is unaffected by scale). We assume $t \neq$ 0 because we have already studied the logarithmic function.

The expected utility for an investment $x$ is:

$$
u_{p}(x)=\frac{(1-p)\left(-1+(1-x)^{t}\right)}{t}+\frac{p\left(-1+(1+R x)^{t}\right)}{t}
$$

The corresponding recurrence using threshold $r$ is:

$$
\bar{U}_{n}(r)=\frac{1}{r+1} \cdot \Psi\left(\frac{r+1}{n}\right)+\frac{r}{r+1} \cdot \bar{U}_{n}(r+1) ; \bar{U}_{n}(n)=\mathcal{U}(1)
$$


Luis Bayón, Pedro Fortuny Ayuso, Jose Maria Grau, Maria del Mar Ruiz, Raquel García-Rubio

and, with the same notation and arguments as before, $g_{n}(x)$ converge on $\left(\frac{1}{R+1}, 1\right)$ to:

$$
g(x):=\frac{1}{t x}\left[-1+\frac{(1+R)^{t}\left((1-x)^{\frac{1}{1-t}}+\left(R^{t} x\right)^{\frac{1}{1-t}}\right)}{\left(R(1-x)^{\frac{1}{1-t}}+(R x)^{\frac{1}{1-t}}\right)^{t}}\right]
$$

and $h_{n}(x)$ converge on $\left(\frac{1}{R+1}, 1\right)$ to:

$$
h(x):=\frac{1}{x}
$$

By Theorem 1, $y(x)$ satisfies in $\left(\frac{1}{R+1}, 1\right)$ the following differential equation:

and also $y(1)=\mathcal{U}(1)$.

$$
y^{\prime}(x)=y(x) h(x)-g(x)
$$

Using Proposition 1, we obtain $\vartheta$ and $y(\vartheta)$. However, the complexity of the differential equation for arbitrary values of $R$ and $t$ prevents the computation of an explicit general solution. For the sake of simplicity, we show, in Table 4, the values of $\vartheta_{R, t}$, and $y\left(\vartheta_{R, t}\right)$, for some $t$ and $R$, which we have computed numerically.

Table 4.Iso-elastic utility function

\begin{tabular}{|c|c|c|c|}
\hline & $R$ & $\vartheta_{R, t}$ & $y\left(\vartheta_{R, t}\right)$ \\
\hline \multirow{5}{*}{$t=1 / 2$} & $1 / 2$ & 0.76873644 & 0.0463451 \\
\cline { 2 - 4 } & 1 & 0.68012743 & 0.125826 \\
\cline { 2 - 4 } & $3 / 2$ & 0.63081156 & 0.210859 \\
\cline { 2 - 4 }$t=-1$ & 2 & 0.59874122 & 0.2952 \\
\hline \multirow{5}{*}{$t=-3$} & $1 / 2$ & 0.78910101 & 0.019017 \\
\cline { 2 - 4 } & 1 & 0.70729857 & 0.0449975 \\
\cline { 2 - 4 } & $3 / 2$ & 0.65972046 & 0.0677138 \\
\cline { 2 - 4 } & 2 & 0.62763302 & 0.086757 \\
\hline \multirow{5}{*}{$t=-5$} & $1 / 2$ & 0.79241027 & 0.0102646 \\
\cline { 2 - 4 } & 1 & 0.70881244 & 0.0228918 \\
\cline { 2 - 4 } & $3 / 2$ & 0.65877942 & 0.0331175 \\
\cline { 2 - 4 } & 2 & 0.62447039 & 0.0412309 \\
\cline { 2 - 4 } & $1 / 2$ & 0.79325493 & 0.0069856 \\
\cline { 2 - 4 } & $2 / 2$ & 0.6576865098 & 0.0152329 \\
\hline
\end{tabular}

As remarked above, for $t=1 / 2$ the function is just an affine translation of $\sqrt{x}$ so that we get the same results (after the suitable scaling) as in Subsection 4.1. 
An Optimal Stopping Problem for an Investing Firm

Isoelasticity guarantees that the optimal ratio of investment $x$ is the same for all levels of wealth.

We finish remarking that the optimal investment, $\mathfrak{m}(p)$ is:

$$
\mathfrak{m}(p)=\left\{\begin{array}{lll}
\frac{-(1-p)^{\frac{1}{1-t}}+(p R)^{\frac{1}{1-t}}}{(1-p)^{\frac{1}{1-t}} R+(p R)^{\frac{1}{1-t}}} & \text { if } & \mathrm{p} \in\left[\frac{1}{1+\mathrm{R}}, 1\right] \\
0 & \text { if } & p \in\left[0, \frac{1}{1+R}\right]
\end{array}\right.
$$

and its associated expected utility for $p>\frac{1}{1+R}$ is:

$$
\begin{gathered}
\Psi(p):=\mathcal{U}_{p}(\mathfrak{m}(p))=\frac{1}{t}\left[(1-p)\left(-1+\left(\frac{1+R}{R+(1-p)^{\frac{1}{-1+t}}(p R)^{\frac{1}{1-t}}}\right)^{t}\right)\right. \\
\left.+p\left(-1+\left(\frac{1+R}{1+(1-p)^{\frac{1}{1-t}} R(p R)^{\frac{1}{-1+t}}}\right)^{t}\right)\right]
\end{gathered}
$$

\section{Conclusions}

Real options has limited applicability in disruptive technological investments and its logic is not applicable in the case of technological investment strategies that generate radical innovations. In this case, the optimal decisions are better represented with the decision model that proposed in this paper. It is broadly derived from the well-known "Secretary Problem"; the decision to accept or reject an option among a sequence is based only on the relative ranks of those investment opportunities considered so far. We present the optimal strategies for several utility functions, also obtaining the optimal amount of capital that the firm must invest. The asymptotic properties of all the models is also considered. As far as we know it is the first study of its kind in the literature.

\section{REFERENCES}

[1] Arikan, A.M., Arikan, I., Kiousis, P. K. (2002), Technological Investment Decisions: Implications for Real Options Logic. Academy of Management, Denver. (2004 version: 8th Annual International Conference, Montreal, Canada);

[2] Bachelier, L. (1900), Théorie de la spéculation. In Annales scientifiques de l'École normale supérieure (Vol. 17, pp. 21-86). tome 17, 21-86;

[3] Bayón, L., Fortuny Ayuso, P., Grau, J.M., Oller-Marcén, A.M., Ruiz, M.M. (2017), The Best-or-Worst and the Postdoc Problems. Journal of Combinatorial Optimization 35(3): 703-723;

[4] Bernardo, A.E., Chowdhry, B. (2002), Resources, Real Options and Corporate Strategy. Journal of Financial Economics 63: 211-234;

DOI: 10.24818/18423264/54.1.20.05 
Luis Bayón, Pedro Fortuny Ayuso, Jose Maria Grau, Maria del Mar Ruiz, Raquel García-Rubio

[5] Black, F., Scholes, M. (1973), The Pricing of Options and Corporate Liabilities. Journal of Political Economy, 81(3): 637-654;

[6] Dixit, A., Pindyck, R. (1994), Investment under Uncertainty. Princeton University Press, Princeton, NJ.;

[7] Dynkin, E.B. (1963), The Optimum Choice of the Instant for Stopping a Markov Process. Soviet Mathematics - Doklad, 4: 627-629;

[8] Ferguson, T.S. (1989), Who Solved the Secretary Problem? Statistical Science, 4(3):282-296;

[9] Ferguson, T. S., Hardwick, J. P., Tamaki, M. (1991), Maximizing the Duration of Owning a Relatively Best Object. Contemporary Mathematics:

Strategies for Sequential Search and Selection in Real Time, American Mathematics Association (T. Ferguson and S. Samuels, eds), 125: 37-58;

[10] Grau Ribas, J.M. (2018), A New Look at the Returning Secretary Problem. Journal of Combinatorial Optimization. 10.1007/s10878-018-0349-8. pp. x-x+21;

[11] Lindley, D.V. (1961), Dynamic Programming and Decision Theory. Journal of the Royal Statistical Society. Series C (Applied Statistics), 10(1): 39-51;

[12] Merton, R.C. (1973), Theory of Rational Option Pricing. Bell Journal of Economics, 4(1): 141-183;

[13] Rust, J. (1996), Numerical Dynamic Programming in Economics.

Handbook of computational economics, 1: 619-729;

[14] Santos, M.S., Vigo-Aguiar, J. (1998), Analysis of a Numerical Dynamic

Programming Algorithm Applied to Economic Models. Econometrica, 66(2): 409-426;

[15] Scott, B. (1999), Computational Methods for the Study of Dynamic

Economies. Oxford University Press;

[16] Szajowski, K.A. (2009), A Rank-Based Selection with Cardinal Payoffs and a Cost Of Choice. Sci. Math. Jpn, 69(2): 285-293;

[17] Vasant, P. (2016), Handbook of Research on Modern Optimization Algorithms and Applications in Engineering and Economics. IGI Global; [18] Vigo-Aguiar, J., Medina, J., Garcia-Rubio, R. (2017), Current Computational Tools for Science Engineering and Economics at CMMSE. Journal of Computational and Applied Mathematics, 318: 1-2. 